\title{
Analysis on Innovation Efficiency of China Meteorological Science and Technology and Its Influencing Factors
}

\author{
Shen Danna ${ }^{1}$, Li Yan $^{2}$ \\ ${ }^{1}$ Development and Research Center, China Meteorological Administration, Beijing, China \\ ${ }^{2}$ School of Statistics and Mathematics, Zhongnan University of Economics and Law, Wuhan, China
}

Email address:

s.dn@163.com (Shen Danna),970654261@qq.com(Li Yan)

\section{To cite this article:}

Shen Danna, Li Yan. Analysis on Innovation Efficiency of China Meteorological Science and Technology and Its Influencing Factors. American Journal of Management Science and Engineering. Vol. 4, No. 2, 2019, pp. 32-38. doi: 10.11648/j.ajmse.20190402.13

Received: April 12, 2019; Accepted: June 5, 2019; Published: June 24, 2019

\begin{abstract}
Based on the meteorological statistics from 2014 to 2017, this paper adopts the DEA-Tobit Two Step method to estimate the innovation efficiency of China meteorological science and technology and then analyses its influencing factors. It is found that during 2014-2017, Beijing has been at the forefront in innovation efficiency of meteorological S\&T, followed by Tianjin. Some other provinces and cities have a decline in technology efficiency. Therefore, pure technology inefficiency still remains a major problem faced by most provinces and cities. Meanwhile, it also reveals that innovation efficiency of meteorological S\&T is significantly and positively impacted by scientific research input and academic structure, but without any significant linear interrelationship with economic development and government influence.
\end{abstract}

Keywords: Innovation Efficiency, Meteorological S\&T, Influencing Factors

\section{Introduction}

As an important measure of efficiency, the scientific and technological innovation capability is attached great importance by foreign institutions to evaluate an institution or a discipline. Generally speaking, there exist few evaluations of innovation capability of meteorological S\&T from a global perspective. Although the Chinese government have expanded investment in S\&T in recent years, it is still unclear what is the innovation efficiency of meteorological S\&T, and whether its development is balanced among different regions, and what are factors essential to improve the efficiency, and what needs further researches.

As the two main evaluation methods for innovation efficiency at present, parametric method is represented by Stochastic Frontier Analysis (SFA) and nonparametric method is represented by Data Envelopment Analysis (DEA) [1]. Chen Xingxing (2016) constructed the efficiency model of China's energy consumption and output and measured the correspond efficiencies of various provinces in China with the SFA. He found that their efficiencies of energy consumption and output were annually decreasing and greatly varied from each other [2]. Chen Yongjun, Zhang
Feilian, Liu Shang (2015) constructed the measurement model of S\&T innovation efficiency of industry, university and research by taking SFA as the method, measured the innovation technology efficiency levels of 25 industry-university-research entities from 2007 to 2014, and meanwhile, analyzed the impact of production through regression analysis [3]. Overseas scholars such as Kohl S, Schoenfelder J, Fügener A, etc. (2018) mainly applied DEA in the healthcare industry and hospital data. They reviewed 262 articles on health care, focused on hospital data and provided important information about DEA settings [4]. DEA analysis framework based on feedback mechanism, Ouenniche J, Carrales S (2018) evaluated the efficiencies of British commercial banks and found that, on average, commercial banks operating in the UK, whether domestic or foreign, had not yet reached acceptable overall technical efficiency, pure technical efficiency and scale efficiency levels [5]. Wolszczak-Derlacz J, Parteka A (2011) used the two-stage DEA method to study the efficiency of European public higher education institutions on account of data from 259 educational institutions in seven European countries from 2001 to 2005. It was found that there were considerable differences in efficiency scores within and among countries. In addition, economies of scale, number and composition of 
colleges, funding sources and gender staff were key factors influencing the efficiency of these institutions [6]. In view of data from 35 countries in 2007 to 2011, Guan J, Zuo K (2014) used the network DEA method to conduct transnational researches on innovation efficiency. They compared the innovation efficiencies of countries, highlighted the weaknesses of innovation efficiency and guided the improvement of innovation efficiency at the national level [7] Chinese scholars such as Fan Hua, Zhou Dequn (2012) applied the reward-variable DEA model to measure the efficiency of Chinese provincial S\&T innovation from 2000-2007, finding that S\&T innovation efficiency was characterized with cyclical fluctuations, and the eastern regions were technologically advanced with higher innovation efficiencies than that of the central and the western regions. However, S\&T efficiencies in the western provinces had increased rapidly, catching up and even surpassing the eastern provinces [8]. Combining with the actual research data and adopting DEA, Zhao Shukuan, Yu Haiqing, Kong Shunlong (2013) evaluated the innovation activities of 151 high-tech enterprises in Jilin province from four aspects: efficiency, effectiveness, scale return and projection analysis. They found that the comprehensive innovation efficiencies of these enterprises were low and greatly varied from each other. The scale efficiency was the main reason leading to the low overall efficiency of innovation [9].

In consideration of the rare researches on the innovation efficiency of meteorological S\&T, the writers of this paper, based on the meteorological statistics of various regions in China from 2014 to 2017, measure the innovation efficiency of meteorological S\&T by applying the DEA model, and then analyze its influencing factors with Tobit model, and finally propose corresponding policies and suggestions to improve the innovation efficiency of meteorological S\&T.

\section{Indicator System and Data Sources}

The S\&T innovation efficiency is the conversion rate of S\&T input and output, namely, the S\&T innovation output obtained from unit innovation input, or the unit innovation input consumed to get the output under the certain technological innovation environment. In brief, S\&T innovation is a complicated system with multiple input and output factors [8]. From an economic point of view, the production Douglas function shows that the input mainly includes capital investment, labor input, and technological progress, and the output is mainly the GDP. In the field of meteorological S\&T, the innovation of that is also a system with multiple input and output factors. Considering the availability of data, we select the total amount of research fund as the capital input and the number of meteorological professional technicians as the labor input, which reflects the investment in S\&T innovation; and we consider the number of SCI papers and research projects and S\&T achievements as the S\&T innovation output, thus to establish the indicator system for the innovation efficiency of meteorological S\&T.
The data involved in this study are all from the China Meteorological Statistics Yearbook in 2014-2017 with the research objects including 31 provinces and cities.

\section{Modelling}

\subsection{DEA Model}

The basic idea of Data Envelopment Analysis (DEA), the method evaluating the relative effectiveness of input-output data [10], is adopting the linear programming method to measure the relative effectiveness of comparable units of the same type on the basis of multiple input and output indicators. DEA and its model were proposed in 1978 by American operations researchers, A. Charnes and W. W. Cooper. This method has been widely used in various industries and sectors, and has great advantages in dealing with multi-indicator inputs and outputs. DEA is expressed as the ratio of output to input, which attempts to maximize the efficiency of the service unit by comparing the efficiency of a particular unit with that of a similar set of units proving the same service. In this process, some units that achieve $100 \%$ efficiency are referred to as relatively effective ones, while others with efficiency scores below $100 \%$ are taken as invalid units. Thus, we can compare the innovation efficiencies of meteorological S\&T in different provinces and cities according to the results of the DEA model, identify relatively inefficient units, measure the severity of inefficiency, and explore ways to reduce inefficiency via the comparison among units.

The reason why DEA model is employed in this paper is that DEA has the obvious advantages in dealing with multi-input and -output problems. Its main performances are as follows: (1) DEA is a non-parametric method. There is no need to know the specific function form of input and output, and to estimate each parameter; (2) The weight in DEA modeling is not subjectively determined by the individual, but calculated by the model according to the optimization criteria to ensure its objectiveness; (3) The evaluation of DEA model is not subject to the measurement units so as to avoid the different results caused by dimensionless methods; (4) DEA model can judge the effectiveness and ineffectiveness of decision-making units, which provides them with quantitative information for improvement [11].

The organization in DEA, generally referred to as the measured performance, is called the decision-making unit (DMU). The most commonly used model is the CCR model that evaluates the techniques and the overall efficiency of DMU. However, CCR model also has its shortcomings. On the premise of the unchanged scale returns and owing to the in-line procession of effects resulted from investment and scale changes, it is impossible to distinguish the influencing factor of the innovation input efficiency and the investment change, namely, the "pure technical efficiency" (PTE) or the scale change, that is, the "scale efficiency" (SE) [12]. As a consequence, this assumption needs to be further relaxed to obtain the BBC model. For all DUMs, the common BBC 
model (based on input orientation) can be shown as:

$$
\begin{gathered}
\min _{\delta, \lambda}\left[\theta_{0}-\varepsilon\left(e^{T} s^{-}+e^{T} s^{+}\right)\right] \\
\text {s.t. }\left\{\begin{array}{c}
\sum_{i=1}^{n} \lambda_{i} y_{i r}-s^{+}=y_{0 r} \\
\sum_{i=1}^{n} \lambda_{i} x_{i j}+s^{-}=\theta x_{0 j} \\
\lambda_{i} \geq 0 ; s^{+} \geq 0 ; s^{-} \geq 0 ; \sum_{i=1}^{n} \lambda_{i}=1 \\
\mathrm{i}=1,2, \ldots, \mathrm{n} ; \mathrm{j}=1,2, \ldots, \mathrm{m} ; \mathrm{r}=1,2, \ldots, \mathrm{s}
\end{array}\right.
\end{gathered}
$$

Among it, $\mathrm{n}$ represents the number of DMU; $\mathrm{m}$ and $\mathrm{s}$ respectively the number of input and out variables; $x_{i j}(j=1,2, \ldots, m)$ and $y_{i r}(r=1,2, \ldots, s)$ respectively the input and the output factor; $s^{-}$and $s^{+}$respectively the slack variable (the difference variable of the input item) and the residual variable (the excess variable of the output item); $\theta_{0}$ the effective of $D M U_{0} ; \varepsilon$ the infinitesimal amount; and $e^{T}=(1, \ldots, 1)^{T}$ [13-14]. In accordance with the optimal solutions $\lambda^{*}, s^{*-}, s^{*+}, \theta^{*}$, the DEA efficiency of DMU can be measured. The conclusions are as follows:

(1) If $\theta^{*}<1, D M U_{j 0}$ is valid for non-DEA;

(2) If $\theta^{*}=1$ and $e^{T} s^{*-}+e^{T} s^{*+}>0, D M U_{j 0}$ is valid for weak DEA;

(3) If $\theta^{*}=1$ and $e^{T} s^{*-}+e^{T} s^{*+}=0, D M U_{j 0}$ is valid for weak DEA;

(4) If $\sum_{j=1}^{m} \lambda_{j}^{*} / \theta^{*}<1$, the scale returns increase;

(5) If $\sum_{j=1}^{m} \lambda_{j}^{*} / \theta^{*}=1$, the scale returns remain unchanged;

(6) If $\sum_{j=1}^{m} \lambda_{j}^{*} / \theta^{*}>1$, the scale returns decrease.

\subsection{Tobit Model}

On the basis of employing the DEA method to obtain the efficiency values of $S \& T$ innovation in various provinces and cities, and in order to further evaluate the impact of the technological innovation environment on the efficiency of S\&T innovation, the authors of this paper choose to use the
Tobit model for analysis. The basic structure of the Tobit model is as follows:

$$
Z_{i}=\left\{\begin{array}{c}
\beta^{T} X_{i}+\varepsilon_{i}, Z_{i}>0 \\
0, Z_{i} \leq 0
\end{array} \quad(\mathrm{i}=1,2, \ldots, \mathrm{n})\right.
$$

Among it, $Z_{i}$ shows the efficiency value; $X_{i}$, the explanatory variable vector; $\beta^{T}$, the parameter vector to be estimated; and $\varepsilon_{i} \sim N\left(0, \sigma^{2}\right)[12]$.

\section{Empirical Analysis}

\subsection{Evaluation of the Innovation Efficiency of \\ Meteorological S\&T Based on DEA Model}

The writers of this paper calculate the S\&T innovation efficiencies of 31 provinces and cities by using Deap2.1 and taking advantage of the input and the output data of meteorological S\&T innovation in these places. During the calculation process, it is found that if the indicators, scientific and technological achievements of some cities value zero, we should process the original data that show zero or negative value in the DEA model. Shen Jiangjian, Long Wen (2015) summarized four methods to deal with the negative output in DEA model and proved the best effect of primary transformation [15]. Even so, in consideration of the great number of zero values of original data involved in this paper and the troublesome primary transformation process, the authors prefer the second method to turn zero values into a small number to process, which also will not change the original result. Hence, 0.01 is selected to get the innovation efficiency values of meteorological S\&T innovation in each

\begin{tabular}{|c|c|c|c|c|c|c|c|c|c|c|c|c|}
\hline \multirow{2}{*}{$\begin{array}{l}\text { Regions } \\
\text { Year }\end{array}$} & \multicolumn{4}{|c|}{ Technical Efficiency (TE) } & \multicolumn{4}{|c|}{ Pure Technical Efficiency (PTE) } & \multicolumn{4}{|c|}{ Scale Efficiency (SE) } \\
\hline & 2014 & 2015 & 2016 & 2017 & 2014 & 2015 & 2016 & 2017 & 2014 & 2015 & 2016 & 2017 \\
\hline Beijing & 1.000 & 1.000 & 1.000 & 1.000 & 1.000 & 1.000 & 1.000 & 1.000 & 1.000 & 1.000 & 1.000 & 1.000 \\
\hline Tianjin & 1.000 & 0.916 & 1.000 & 1.000 & 1.000 & 1.000 & 1.000 & 1.000 & 1.000 & 0.916 & 1.000 & 1.000 \\
\hline Hebei & 0.580 & 0.889 & 1.000 & 0.945 & 0.590 & 0.900 & 1.000 & 0.968 & 0.983 & 0.988 & 1.000 & 0.976 \\
\hline Shanxi & 0.563 & 0.853 & 0.689 & 1.000 & 0.732 & 0.898 & 0.708 & 1.000 & 0.769 & 0.950 & 0.973 & 1.000 \\
\hline Inner Mongolia & 0.440 & 0.345 & 0.611 & 0.450 & 0.447 & 0.375 & 0.749 & 0.466 & 0.984 & 0.919 & 0.816 & 0.966 \\
\hline Liaoning & 1.000 & 0.843 & 1.000 & 0.752 & 1.000 & 0.853 & 1.000 & 0.754 & 1.000 & 0.988 & 1.000 & 0.998 \\
\hline Jilin & 0.491 & 0.985 & 1.000 & 1.000 & 0.806 & 1.000 & 1.000 & 1.000 & 0.608 & 0.985 & 1.000 & 1.000 \\
\hline Heilongjiang & 0.345 & 1.000 & 1.000 & 0.919 & 0.665 & 1.000 & 1.000 & 0.922 & 0.519 & 1.000 & 1.000 & 0.997 \\
\hline Shanghai & 0.973 & 1.000 & 0.784 & 0.894 & 1.000 & 1.000 & 0.794 & 0.897 & 0.973 & 1.000 & 0.987 & 0.996 \\
\hline Jiangsu & 0.575 & 0.800 & 1.000 & 1.000 & 0.676 & 0.802 & 1.000 & 1.000 & 0.850 & 0.998 & 1.000 & 1.000 \\
\hline Zhejiang & 0.695 & 1.000 & 0.738 & 0.992 & 0.695 & 1.000 & 1.000 & 1.000 & 1.000 & 1.000 & 0.738 & 0.992 \\
\hline Anhui & 0.514 & 0.629 & 0.749 & 0.903 & 0.551 & 0.676 & 0.767 & 0.908 & 0.934 & 0.930 & 0.977 & 0.995 \\
\hline Fujian & 0.553 & 1.000 & 1.000 & 1.000 & 0.797 & 1.000 & 1.000 & 1.000 & 0.694 & 1.000 & 1.000 & 1.000 \\
\hline Jiangxi & 1.000 & 0.474 & 0.801 & 1.000 & 1.000 & 0.499 & 0.801 & 1.000 & 1.000 & 0.950 & 1.000 & 1.000 \\
\hline Shandong & 1.000 & 0.633 & 1.000 & 0.296 & 1.000 & 0.704 & 1.000 & 0.533 & 1.000 & 0.899 & 1.000 & 0.555 \\
\hline Henan & 1.000 & 0.342 & 0.455 & 0.728 & 1.000 & 0.659 & 0.750 & 0.736 & 1.000 & 0.518 & 0.607 & 0.989 \\
\hline Hubei & 0.901 & 1.000 & 0.754 & 0.981 & 1.000 & 1.000 & 0.754 & 1.000 & 0.901 & 1.000 & 0.999 & 0.981 \\
\hline Hunan & 0.318 & 0.677 & 0.919 & 0.782 & 0.346 & 0.678 & 0.928 & 0.790 & 0.918 & 1.000 & 0.990 & 0.989 \\
\hline Guangdong & 0.643 & 1.000 & 1.000 & 1.000 & 0.649 & 1.000 & 1.000 & 1.000 & 0.990 & 1.000 & 1.000 & 1.000 \\
\hline Guangxi & 0.492 & 1.000 & 0.596 & 0.796 & 0.532 & 1.000 & 0.616 & 0.857 & 0.924 & 1.000 & 0.967 & 0.928 \\
\hline Hainan & 0.650 & 1.000 & 0.854 & 0.482 & 0.749 & 1.000 & 0.873 & 0.827 & 0.868 & 1.000 & 0.978 & 0.583 \\
\hline Chongqing & 0.409 & 0.403 & 0.611 & 0.574 & 0.592 & 0.603 & 0.842 & 0.778 & 0.691 & 0.669 & 0.725 & 0.738 \\
\hline
\end{tabular}
province and city. The results are shown in Table 1.

Table 1. Innovation Efficiency Values of Meteorological S\&T in Various Provinces and Cities (2014-2017). 


\begin{tabular}{lllllllllllll}
\hline Regions & \multicolumn{3}{l}{ Technical Efficiency (TE) } & \multicolumn{4}{c}{ Pure Technical Efficiency (PTE) } & \multicolumn{3}{l}{ Scale Efficiency (SE) } \\
\hline Year & $\mathbf{2 0 1 4}$ & $\mathbf{2 0 1 5}$ & $\mathbf{2 0 1 6}$ & $\mathbf{2 0 1 7}$ & $\mathbf{2 0 1 4}$ & $\mathbf{2 0 1 5}$ & $\mathbf{2 0 1 6}$ & $\mathbf{2 0 1 7}$ & $\mathbf{2 0 1 4}$ & $\mathbf{2 0 1 5}$ & $\mathbf{2 0 1 6}$ & $\mathbf{2 0 1 7}$ \\
\hline Sichuan & 0.482 & 0.682 & 1.000 & 1.000 & 0.487 & 0.759 & 1.000 & 1.000 & 0.991 & 0.899 & 1.000 & 1.000 \\
Guizhou & 0.456 & 1.000 & 1.000 & 0.910 & 0.663 & 1.000 & 1.000 & 0.920 & 0.687 & 1.000 & 1.000 & 0.989 \\
Yunnan & 0.680 & 0.725 & 0.453 & 0.280 & 0.873 & 0.788 & 0.541 & 0.375 & 0.779 & 0.920 & 0.838 & 0.746 \\
Tibet & 0.244 & 0.440 & 0.764 & 0.250 & 0.738 & 1.000 & 1.000 & 0.472 & 0.330 & 0.440 & 0.764 & 0.530 \\
Shaanxi & 0.518 & 0.769 & 0.301 & 0.617 & 0.618 & 0.805 & 0.305 & 0.623 & 0.837 & 0.955 & 0.987 & 0.989 \\
Gansu & 0.926 & 0.388 & 0.423 & 0.731 & 0.988 & 0.402 & 0.428 & 0.810 & 0.937 & 0.964 & 0.989 & 0.903 \\
Qinghai & 0.433 & 0.492 & 0.875 & 0.621 & 0.774 & 0.596 & 0.876 & 0.631 & 0.559 & 0.825 & 0.999 & 0.984 \\
Ningxia & 0.543 & 0.715 & 1.000 & 1.000 & 1.000 & 1.000 & 1.000 & 1.000 & 0.543 & 0.715 & 1.000 & 1.000 \\
Xinjiang & 0.488 & 0.597 & 0.869 & 0.919 & 0.495 & 0.597 & 1.000 & 1.000 & 0.987 & 0.999 & 0.869 & 0.919 \\
Average Value & 0.642 & 0.761 & 0.814 & 0.801 & 0.759 & 0.828 & 0.861 & 0.848 & 0.850 & 0.913 & 0.939 & 0.927 \\
\hline
\end{tabular}

Innovation efficiency evaluation mainly includes comprehensive technical efficiency, pure technical efficiency and scale efficiency. And pure technical efficiency reflects the production efficiency of the DMU at the optimal scale under certain input factors; while scale efficiency presents the gap between actual and the optimal scale. It is generally believed that the pure technical efficiency times scale efficiency is comprehensive technical efficiency. What's more, the comprehensive technical efficiency is an overall measurement and evaluation of the resource allocation ability and resource utilization efficiency of DMUs; the pure technical efficiency shows the production efficiency influenced by management and technology and other factors; and scale efficiency presents the production efficiency affected by the scale. The comprehensive technical efficiency value is 1 , indicating the integrated and effective input and output of DMUs, that is to say, units under inspection achieve both technical scale efficiency simultaneously. But if the pure technical efficiency value is 1 , it proves the effectiveness in utilization of the input resource at the current decision-making level. And the fundamental reason for failing to achieve comprehensive effectiveness lies in the invalid scale.

It can be seen from Table 1 that the comprehensive technical efficiencies of Beijing, Tianjin, Liaoning, Jiangxi, Shandong and Henan in 2014 were effective, taking the frontier in meteorological S\&T innovation system. Besides, Shanghai, Zhejiang, Hubei, Guangdong, Hainan, Yunnan and Gansu exceeded the national average value of comprehensive technical efficiency and maintained a relatively stable trend. From vertical perspective, there is a certain growth in the comprehensive technical efficiency level of various provinces and cities in 2014-2017 with average values of $0.642,0.761$, $0.814,0.801$, which means that on the premise of same output, the meteorological S\&T departments of various provinces and cities in China have a potential of saving from $35.8 \%$ in 2014 to $19.9 \%$ in 2017 , further indicating the saving in meteorological S\&T investment in terms of the national average. In the meantime, both the pure technical efficiency and scale efficiency are greatly promoted, respectively from 0.759 in 2014 to 0.848 in 2017 and from 0.850 in 2014 to 0.927 in 2017.

Meanwhile, it also can be seen from Table1 that the effective DEA units in 2014 included: Beijing, Tianjin, Liaoning, Jiangxi, Shandong and Henan; in 2015 included: Beijing, Heilongjiang, Shanghai, Zhejiang, Fujian, Hubei, Guangdong, Guangxi, Hainan and Guizhou; in 2016 included:
Beijing, Tianjin, Hebei, Liaoning, Jilin, Heilongjiang, Jiangsu, Fujian, Shandong, Guangdong, Sichuan, Guizhou and Ningxia; in 2017 included: Beijing, Tianjin, Shanxi, Jilin, Jiangsu, Fujian, Jiangxi, Guangdong, Sichuan and Ningxia. However, Inner Mongolia, Anhui, Hunan, Chongqing, Yunnan, Tibet, Shaanxi, Gansu, Qinghai and Xinjiang are all non-DEA effective units during these years and all with obvious fluctuations in values of the comprehensive technical efficiency, pure technical efficiency and scale efficiency, which indicates their relatively larger input but lower efficiency compared with that of other provinces and cities under a certain output condition. Further analysis found that in terms of interprovincial regions, these three efficiencies of Beijing have remained at the level of 1 from 2014 to 2017, which shows that compared with other regions, Beijing has achieved the minimal input in meteorological S\&T, taking the best production frontier for innovation efficiency of meteorological S\&T. In addition, the efficiency values of Tianjin over the years are all above 0.9 , which is very close to the production frontier. And its average value of comprehensive technical efficiency has reached 0.979 with a slight decline in 2015, keeping Tianjin occupying the best production frontier in other years.

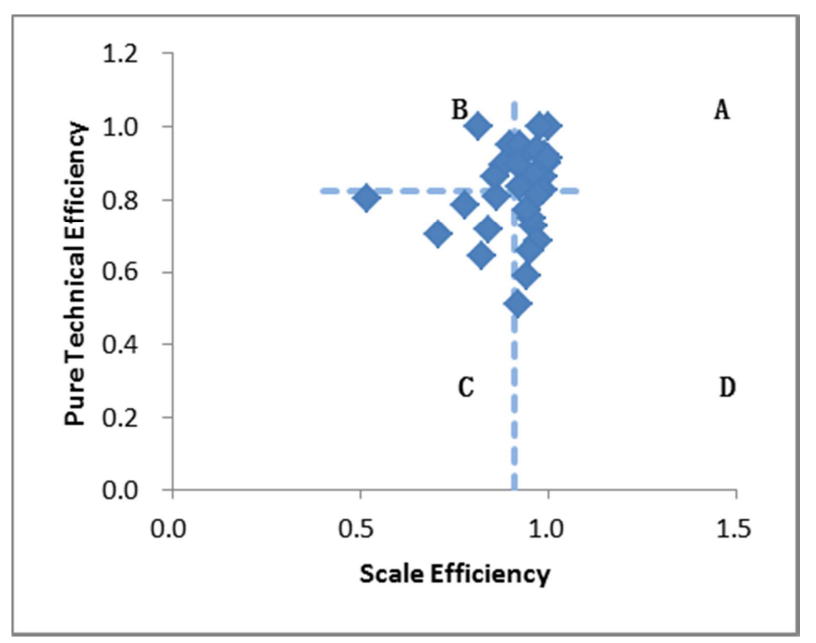

Figure 1. The Average Value Decomposition of the Static Efficiency of Various Provinces and Cities in 2014-2017.

Figure 1 demonstrates the average value decomposition of the static efficiency of various provinces and cities in 2014-2017. Owing to the dense distribution of provinces and cities, it doesn't show the names of the specific provinces and 
cities with specific points. Just the corresponding analysis is carried out. In this figure, the horizontal axis means the scale efficiency and the vertical axis means the pure technical efficiency, and the dotted line means the pure technical efficiency and the scale efficiency represented by the national average of technological innovation efficiency in 2014-2017. Correspondingly, provinces and cities are divided into four categories: A, B, C and D. Region A includes Beijing, Tianjin, Hebei, Shanxi, Liaoning, Shanghai, Jiangsu, Zhejiang, Fujian, Jiangxi, Hubei, Guangdong and Guizhou, nearly occupying $42 \%$ of all provinces and cities in China, which is the most concentrated distribution; Region B with lower scale efficiency includes Jilin, Heilongjiang, Hainan and Ningxia; Region $\mathrm{C}$ with lower scale efficiency and pure technical efficiency includes Shandong, Henan, Chongqing, Yunnan, Tibet and Qinghai; Region D with lower pure technical efficiency includes Inner Mongolia, Anhui, Hunan, Guangxi, Sichuan, Shaanxi, Gansu and Xinjiang. Therefore, it is not difficult to find that provinces belonging to Region B and D account for $38.71 \%$ of all national provinces, indicating that there is a skew in the technical efficiency among some provinces and cities; provinces in Region C and D take up $45.16 \%$ of all national provinces, which explains that the technology inefficiency remains a major problem confronted by most provinces and cities, especially for provinces and cities in Region C; provinces in Region A and D account for $67.74 \%$ of all national provinces, implying that the majority of provinces perform well in scale efficiency.

The annually comprehensive technical efficiency as well as the variable coefficient of decomposition items are calculated in order to investigate the differences in technical efficiency among provinces, as shown in Figure 2. As can be seen from this figure, within the research scope, the variable coefficient of the overall technical efficiency and the scale technical efficiency both began to decline from 2014, and then to rise after 2016. Besides, both the increase and the decline rate of the variable coefficient of the comprehensive technical efficiency are greater than that of the scale efficiency, which manifests the gradually shrinking differences in the comprehensive technical efficiency and the scale technical efficiency of the meteorological S\&T innovation among provinces in 2014-2016. However, after 2016, the difference gap is further expanded, and the difference in the overall technical efficiency is greater than that in the scale of technical

$$
Z_{i}=\beta_{0}+\beta_{1} X_{1 i}+\beta_{2} X_{2 i}+\beta_{3} X_{3 i}+\beta_{4} X_{4 i}+\varepsilon_{i}(i=1,2, \ldots, 31)
$$

Among it, $Z_{i}$ represents the efficiency value of the $i$-th province; $X_{1 i}$, the economic development status; $X_{2 i}$, the scientific research input; $X_{3 i}$, the academic structure; $X_{4 i}$, the government influence; $\varepsilon_{i}$, the random interference term obeying the normal distribution.

Using the software, stata14.0, to fit the data, we can obtain the Tobit model of the influencing factors of meteorological S\&T innovation efficiency in 31 provinces and cities. Results are shown in Table 2. efficiency. Relatively speaking, there are almost no major changes in the variable coefficient of pure technical efficiency. That is to say, there have been no major changes in the ability of a given input to achieve maximum output in recent years.

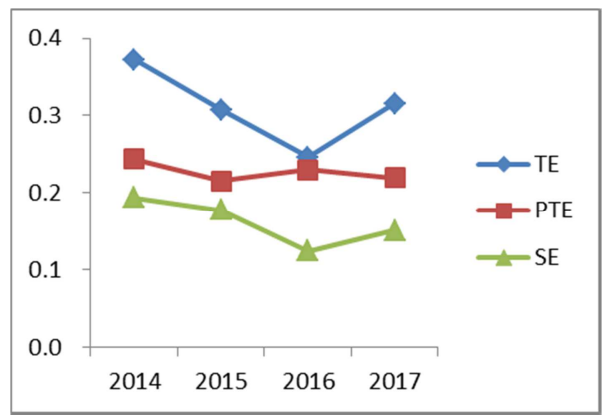

Figure 2. The Variable Coefficient of Decomposition and the Static Efficiency Value of Various Provinces and Cities in 2014-2017.

\subsection{Analysis of Influencing Factors of Meteorological S\&T Innovation Efficiency Based on Tobit Model}

The analysis of the influencing factors of innovation efficiency focuses on the role of key elements played in improving innovation efficiency in innovation system [16]. For the meteorological field, this paper takes the influences of economic development, scientific research input, academic structure, and government influence on the meteorological S\&T innovation efficiency into consideration [8]. And what's more, in view of the data availability, the economic development status is expressed by the growth rate of GDP in each region, and the scientific research input is showed by the growth rate of the total amount of funds in each region. The academic structure is represented by the proportion of graduates with master's degree or above in each region and the government influence is conveyed by the growth rate of the amount of S\&T funds issued by the local government.

Combined with the actual situation of this paper, since the efficiency value of multiple DMUs in the dependent variable is 1 , within the efficiency boundary of DEA and in the case where such a plurality of samples become a certain limit value within a specific range, the conventional method can't be used to explain the nature difference between the limit value and the non-limit value. Hence, we confine the limit value of DEA efficiency, 1, and then create the following Tobit model:
Table 2. Tobit Regression Results of the Influencing Factors of Meteorological S\&T Innovation Efficiency in Various Regions.

\begin{tabular}{llll}
\hline Variable & Coefficient & Standard Error & P Value \\
\hline$\beta_{0}$ & $0.6168^{* * *}$ & 0.0757 & 0.000 \\
$\beta_{1}$ & 0.6748 & 0.5572 & 0.226 \\
$\beta_{2}$ & $0.0671^{* *}$ & 0.0294 & 0.022 \\
$\beta_{3}$ & $0.9418^{* * *}$ & 0.3274 & 0.004 \\
$\beta_{4}$ & -0.0857 & 0.1684 & 0.611 \\
\hline
\end{tabular}

Note: $* * *$ indicates the significance level at $1 \% ; * *$ indicates the significance level at $5 \%$; indicates the significance level at $10 \%$. 
Although the Tobit model can't directly explain the influence between the dependent variable and the independent variable as the ordinary least square method does, the sign of the estimated coefficient is consistent with the partial effect [8]. Therefore, it can be seen from the above results that within the scope of studied sample, the regional meteorological S\&T innovation efficiency has a significant positive correlation with the scientific research input and academic structure of each region, while there's no linear relationship between the economic development status and the government influence and the meteorological S\&T innovation. Specially, the impact of scientific research input on the innovation efficiency of meteorological S\&T in various regions is significantly positive, that is, the greater the investment in scientific research, the higher the efficiency. What's more, the influence of academic structure on the innovation efficiency of meteorological S\&T in each region is significantly positive, that is, the more highly educated talents, the higher the efficiency. As the basis of human resource investment in scientific and technological innovation, highly educated talents exert a significant effect on the development of meteorological S\&T innovation efficiency.

\section{Conclusions and Expectations}

In this paper, the DEA-Tobit two-step method is used to study the innovation efficiency of meteorological S\&T and its influencing factors in 31 provinces and cities from 2014 to 2017. The following preliminary results can be obtained from the DEA model: expect for the slight decline in 2015, Beijing had been at the forefront in terms of innovation efficiency of meteorological S\&T in 2014-2017, followed by Tianjin, while Inner Mongolia, Anhui, Hunan, Chongqing, Yunnan, Tibet, Shaanxi, Gansu, Qinghai and Xinjiang were all non-EDA effective units during these years. In recent years, under certain conditions, these non-DEA effective units increase more investment than that of other provinces and cities, which results in the relatively low efficiency.

Meanwhile, based on the average decomposition of the static efficiency of meteorological S\&T innovation, the authors of this paper find that Jilin, Heilongjiang, Hainan and Ningxia have low scale efficiencies, and Inner Mongolia, Anhui, Hunan, Guangxi, Sichuan, Shaanxi, Gansu and Xinjiang have low pure technical efficiencies. Pure technical inefficiency remains a major problem faced by most provinces and cities, especially those provinces and cities with lower pure technical efficiency and scale efficiency, such as Shandong, Henan, Chongqing, Yunnan, Tibet and Qinghai. However, generally speaking, most provinces and cities are with better scale efficiency, including Beijing, Tianjin, Hebei, Shanxi, Liaoning, Shanghai, Jiangsu, Zhejiang, Fujian, Jiangxi, Hubei, Guangdong, Guizhou, Inner Mongolia, Anhui, Henan, Guangxi, Sichuan, Shaanxi, Gansu and Xinjiang.

Further analysis of the factors affecting the innovation efficiency of meteorological S\&T shows that the main influencing factors are scientific research investment and academic structure. These two factors are in significantly positive correlations. The investment in scientific research in this paper is expressed by the growth rate of the funds in each region. And the academic structure is represented by the proportion of masters and above in the employment team. Eventually, it shows that the more funds are invested in various regions and the more highly educated talents are involved, the higher is the innovation efficiency of meteorological S\&T. Which is also consistent with our perception. However, it is worth mentioning that within the research scope of this paper, there is no significant relationship between the innovation efficiency of meteorological S\&T in various regions and the economic development and the government influence. The economic development is expressed by the GDP growth rate of each region, and the government influence is shown by the growth rate of S\&T funds issued by the local governments. Theoretically, the more a region's economy is developed, the greater attention the government will attach to the meteorological S\&T innovation. And eventually, the higher is the innovation efficiency of meteorological, which is not consistent with the conclusions drawn in this paper. But it must be noted that the Tobit model used in this paper only reflects the linear correlation between innovation efficiency of meteorological S\&T and the influencing factors considered, and other non-linear correlations will not be further discussed in this paper.

Some corresponding suggestions can be made according to the above conclusions. For non-DEA effective units, such as Inner Mongolia, Anhui, Hunan, Chongqing, Yunnan, Tibet, Shaanxi, Gansu, Qinghai and Xinjiang, they should increase their investment in scientific research, introduce highly educated talents, guarantee the maximum output with minimum input, draw close to the production frontier, and strive to be an effective unit of DEA. Then for regions with low efficiency of pure technology, including Inner Mongolia, Anhui, Hunan, Guangxi, Sichuan, Shaanxi, Gansu and Xinjiang, it is necessary to take full advantage of resources and endeavor to achieve maximum output with certain input. In addition, for areas with lower scale efficiency, like Jilin, Heilongjiang, Hainan and Ningxia, they should re-consider and re-confirm their investment scale, as the previous large scale of investment brought them rather lower output.

Innovation efficiency is the basic indicator for building innovative countries and regions. And innovation in all walks of life plays a significant role in promoting regional development in the new era. Though meteorological S\&T innovation is a rather new field, this paper comes to some meaningful conclusions via empirical analysis. However, it is also noted that there are still various problems to be further concerned and studied in the meteorological field.

\section{References}

[1] Zhang Yuting, Yang Hualing. (2018). An Overview of Evaluation Methods of Technological Innovation Efficiency. China Management Informatization, (4), 82-84. 
[2] Chen Xingxing. (2016). Measurement and Analysis of China's Energy Consumption and Output Efficiency. Statistics \& Decision, (23), 114-119.

[3] Chen Yongjun, Zhang Feilian, Liu Shang. (2015). Research on Technological Innovation Efficiency of Industry-University-Research Institute Based on Stochastic Frontier Analysis. Science \& Technology Progress and Policy, (24), 21-24.

[4] Kohl S, Schoenfelder J, Fügener A, et al. (2018). Correction to: The use of Data Envelopment Analysis (DEA) in healthcare with a focus on hospitals. Health Care Management Science, (15), $1-1$.

[5] Ouenniche J, Carrales S. (2018). Assessing efficiency profiles of UK commercial banks: a DEA analysis with regression-based feedback. Annals of Operations Research, (1), 1-37.

[6] Wolszczak-Derlacz J, Parteka A. (2011). Efficiency of European public higher education institutions: a two-stage multicountry approach. Scientometrics, (89), 887-917.

[7] Guan J, Zuo K. (2014). A cross-country comparison of innovation efficiency. Scientometrics, 100 (2): 541-575.

[8] Fan Hua, Zhou Dequn. (2012). Regional Science and Technology Innovation Efficiency Evolution and Its Affect Factors in Chinese Provinces. Science Research Management, 33 (1): 10-18.

[9] Zhao Shukuan, Yu Haiqing, Gong Shunlong. (2013). The Innovation Efficiency of Hi-tech Enterprises in Jilin Province Based on DEA Method. Science Research Management, 34 (2), 36-43.
[10] Yang Guoliang, Liu Wenbin, Zheng Haijun. (2013). Review of Data Envelopment Analysis. Journal of Systems Engineering, 28 (6), 840-860.

[11] Wang Tingting. (2013). Efficiency Measurement of Interprovincial Energy Based on DEA and FDA Methods in China. Tsinghua University Press.

[12] Huang Funing. (2013). Evaluation of ChiNext Innovation Efficiency. Economy \& Management Publishing House.

[13] Chen Bing, Ji Shengbao. (2013). The Performance Evaluation of Listed Chinese Pharmaceutical Companies and the Influencing Factors: An Empirical DEA-Tobit Evidence Based on the Panel Data. Journal of Central University of Finance \& Economics, 1 (8).

[14] Chen Xiaowei. (2011). Study on Efficiency Evaluation and Its Influencing Factors of Chinese Commercial Banks. Southwest Jiaotong University Press.

[15] Shen Jiangjian, Long We. (2015). Treatment of Negative Output in DEA Model-Based on the Application of Software DEAP. Hefei: Chinese Academy of Management.

[16] Guo Danbo, Lei Jiaxiao, Zhang Junfang, etc. (2012). Research on the Efficiency and Influencing Factors of National Innovation System-Based on DEA-Tobit Two-Step Analysis. Journal of Tsinghua University (Philosophy and Social Sciences), (2), 142-150. 